\title{
Una integración dividida: las múltiples iniciativas que generan dificultades
} para lograr una integración regional en Latinoamérica

\author{
An integration divided: the many initiatives \\ that create difficulties to achieve regional \\ integration in Latin America
}

\author{
Julio César Botero Robayo (Colombia) \\ Magíster en Asuntos Internacionales \\ Institución Universitaria Politécnico Grancolombiano \\ jcbotero@poligran.edu.co
}

Recibido: 25 de abril de 2013

Evaluado: 26 de junio de 2013

Aceptado: 30 de junio de 2013

\section{Resumen}

En Suramérica existen bloques subregionales de comercio o foros políticos continentales, pero no hay todavía ningún espacio que pueda ser considerado como una unión económica. Dichos bloques comerciales en la región deben ser estudiados con especial detenimiento, puesto que sus implicaciones sobre la economía regional pueden ser negativas. La CAN, el Mercosur y la recientemente creada Alianza del Pacífico, son los tres espacios de integración comercial en la región. Tanto en el interior como en el exterior de dichos bloques se pueden percibir beneficios de diferente naturaleza, sin embargo las relaciones entre ellos es prácticamente nula. Ese hecho, en lugar de fortalecer la conjunción continental, puede implicar no una ruptura, pero, sí una clara división en el interior de la economía suramericana.

Palabras clave: integración, globalización, Latinoamérica, comercio, Mercosur.

\begin{abstract}
In South America there are sub-regional trading blocs or continental political forums, but there is still no space that can be considered as an economic union. These trading blocs in the region should be studied with special attention, since its implications on the regional economy can be negative. The CAN, Mercosur and the newly formed Pacific Alliance, are the three areas of trade integration in the region. Both inside and outside of said blocs benefits of different nature can be perceived, however the relationship between them is negligible. This fact, rather than strengthening continental integration may involve perhaps not a break, but a clear division within the South American economy.
\end{abstract}

Keywords: integration, globalization, Latin America, trade, Mercosur. 


\section{Introducción}

Son múltiples los esfuerzos por integrar las economías suramericanas. Desde tiempos de Bolívar se planteaba la necesidad de la unidad suramericana como un elemento clave para su independencia y desarrollo. Han pasado dos siglos y todavía no existe una estructura sólida que cohesione las economías de la región.

El objetivo del presente escrito radica en realizar una investigación descriptiva de los resultados de los esfuerzos en materia de integración económica, política y social de Latinoamérica. Asimismo, se espera que en el futuro próximo surja en la institución un semillero de investigación relacionado con el tema, con el fin de motivar a los estudiantes a explorar los temas de actualidad regional.

Retomando las iniciativas de hace ya más de dos siglos esbozadas por Bolívar, O”Higgins y San Martín, quienes exponían sus ideas integradoras: "De la unión y cordialidad recíproca depende el próspero destino de las Américas y la división solo puede producir miserias y lágrimas" (O"Higgins, s. f.); "Divididos seremos esclavos, unidos venceremos" (San Martín); y "Para nosotros la patria es América" (Bolívar, 1814), ver: Moreno (2007), en 2004 uno de los hombres más poderosos del planeta, el señor Hu Jintao, en una gira oficial del Gobierno chino por el mundo, se presentó frente al Parlamento de Brasil con una propuesta particular y, hasta ese día inesperada: realizar una serie de esfuerzos en materia de estrechar los lazos de las economías China y Latinoamericana: "We both sides should seize opportunities and work together to drive the China-Latin America friendly cooperation to increasingly new levels"1 (Hu, 2004).

\section{Una integración dividida}

No es desde el corazón latinoamericano que vuelve a surgir una idea integradora, sino, por el contrario, su nacimiento se registra desde el otro extremo del océano Pacífico. Al parecer, el señor $\mathrm{Hu}$ conoce las necesidades económicas del continente americano mejor que los latinoamericanos, o por lo menos, ha leído lo que hace ya algún tiempo desarrolló un teórico económico de la región.

Luego de medio siglo del planteamiento de los postulados de Raúl Prebisch (1959) en la Comisión Económica para América Latina y el Caribe (Cepal), relacionados con la industrialización y la necesidad de una integración de mercados en América Latina, es lamentable ver la actual situación del mercado regional.

Como lo menciona el economista chileno Roberto Pizarro, los resultados de los primeros esfuerzos integracionistas son decepcionantes:

No solo porque las exportaciones intrarregionales apenas alcanzan $15 \%$ del total de lo que nuestros países transan en el mercado mundial, sino también porque las acciones concretas a favor de la unión regional se han oscurecido, al tiempo que la retórica se acrecienta y las controversias se multiplican,

1 "Ambas partes deben aprovechar las oportunidades y trabajar juntas para impulsar la cooperación amistosa entre China y América Latina hacia nuevos niveles" 
con preocupantes distanciamientos diplomáticos (Pizarro, 2008, p. 214).

En América Latina y el Caribe se han intentado acuerdos regionales desde hace por lo menos sesenta años. Se pueden reconocer por lo menos tres fases: en la década de los cincuenta se lanzaron propuestas como la Asociación Latinoamericana de Libre Comercio (Alalc) y el Mercado Común Centroamericano (MCCA); en la décadas de los sesenta se conformaron el Pacto Andino y la Comunidad del Caribe (Caricom); finalmente, desde mediados de la década de los ochenta se observa la reconfiguración de la integración andina hacia la Comunidad Andina (CAN), y luego el Tratado de Libre Comercio de América del Norte (TLCAN) y el Mercado Común del Sur (Mercosur) (Gudynas, 2007).

¿Qué ha sucedido para que los procesos integradores latinoamericanos no dieran los resultados esperados?

En ese contexto, es necesario revisar los diferentes experimentos integradores subregionales en Latinoamérica, enfocándonos en Suramérica. Lo anterior, debido a motivos pragmáticos en cuanto al tiempo y a los recursos destinados para el presente estudio y a razones discrecionales del investigador. Los principales objetivos de los diferentes acuerdos de integración económica subregional en América Latina, hasta la fecha, son los siguientes.

La primera iniciativa de integración fue la Alalc, la cual daba un plazo de doce ańos para la creación de una zona de libre comercio, es decir que en 1972 ya debería de estar en desarrollo. Sin embargo, eso nunca sucedió (Alalc, 1960). Por el contrario, al ver que no produjo ningún resultado, en 1980, en el ámbito del
Tratado de Montevideo, fue constituida la Asociación Latinoamericana de Integración (Aladi), que, a diferencia de su predecesora, es un espacio más político que comercial.

El otro intento por integrar las economías de la región nacido en los años sesenta fue el Mercado Común Centroamericano (MCCA), una iniciativa de Guatemala, Honduras, Nicaragua, El Salvador y Costa Rica. Si bien su tamaño no es tan significante dentro de la región, es una experiencia exitosa, tanto en el interior de la región como en las relaciones comerciales con economías de otras latitudes; por ejemplo, cuentan con un tratado interregional de comercio con la Unión Europea (UE).

A finales de los años sesenta, en 1969, con el Acuerdo de Cartagena se crea la CAN, un espacio político en el cual se trabaja para "alcanzar un desarrollo integral, más equilibrado y autónomo, mediante la integración andina" (CAN, 1969, p. 1). Más adelante serán dedicadas unas líneas adicionales para explicar la dinámica de este acuerdo.

Cuatro años más tarde, se crea la Caricom, cuyos principales objetivos eran: promover la integración económica y la cooperación entre sus miembros, asegurar la distribución equitativa de los beneficios de la integración y coordinar su política exterior (Caricom, 1973).

Con las bases, o por lo menos con la intención de establecer zonas de libre comercio en el Caribe, en Centro y Suramérica, se plantea por primera vez, a mediados de los setenta, una conjunción de toda la región. El Sistema Económico Latinoamericano y del Caribe (SELA), por su parte, es un organismo consultivo regional constituido en 1975 cuyo fin es el promover la cooperación intrarregional 
para acelerar el desarrollo económico y social de todos sus miembros (SELA, 1975).

Como se mencionó, surge una asociación de integración, la Aladi, en respuesta a las fallas y a la inoperatividad de la Alalc. La Aladi se constituyó en Montevideo en 1980 y es una asociación que promueve el pluralismo en materia política y económica, además de que busca "la convergencia progresiva de acciones parciales hacia la formación de un mercado común latinoamericano", a la vez que nuevos instrumentos para la profundización del comercio regional (Aladi, 1980, p. 1).

Llega la década de los noventa y con ella uno de los más exitosos modelos de integración económica en el continente: el Mercosur, creado en 1991, en el Tratado de Asunción, y sus principales objetivos son:

La libre circulación de bienes, servicios y factores productivos entre países, el establecimiento de un arancel externo común y la adopción de una política comercial común, la coordinación de políticas macroeconómicas y sectoriales entre los Estados partes y la armonización de las legislaciones para lograr el fortalecimiento del proceso de integración (Mercosur, 1991, p. 1).

En el Caribe se percibe que es necesario fortalecer la Caricom y darle un soporte político más sólido, ir más allá del comercio, dar un paso adelante. En 1994 se crea la AEC-ACS, Asociación de Estados del Caribe, su objetivo: "El fortalecimiento de la cooperación regional y del proceso de integración, con el propósito de crear un espacio económico ampliado en la región" (AEC, 1994, p. 1).
A manera de copia de las intenciones del SELA, surge otra iniciativa integradora regional, pero esta vez de alcance hemisférico, al pretender incluir a las economías de toda América, desde Canadá y Estados Unidos hasta Argentina y Chile, incluyendo al Caribe, a la Región Andina, a Centroamérica y, por supuesto, a México y Brasil. Sin embargo, el Área de Libre Comercio de las Américas (ALCA), no se ha puesto en marcha. Desde la primera reunión en Miami, han transcurrido múltiples reuniones de ministros y dignatarios, como todas las anteriores, infructuosas. Al día de hoy, el ALCA sigue siendo una iniciativa de "unir las economías de las Américas en una sola área de libre comercio" (ALCA, 1994, p. 1).

Con la llegada del nuevo milenio y con él una nueva propuesta sociopolítica, el "socialismo del siglo XXI", se crea el ALBA (Alianza Bolivariana para los Pueblos de Nuestra América). Esta es la iniciativa gestada por el expresidente Hugo Chávez, la cual, en sus palabras: "es el proyecto histórico de Simón Bolívar de unidad de la Patria Grande, para garantizar al pueblo la mayor suma de felicidad posible, la mayor suma de seguridad social y la mayor suma de estabilidad política" (ALBA, 2004, p. 1).

La Unión de Naciones Suramericanas (Unasur), es un organismo internacional creado en 2008 para: "Construir, de manera participativa y consensuada, un espacio de integración y unión en lo cultural, social, económico y político entre sus pueblos" (Unasur, 2008, p. 1). Es otro foro de contenidos políticos más que cualquier otra cosa; un espacio en el que los presidentes de Suramérica se reúnen para debatir temas de seguridad y tratar de resolver las disputas o inconvenientes del devenir regional. No es mucho lo que 
este espacio aporta al comercio, salvo que puede ser el espacio donde confluyen los intereses tanto del Mercosur como de la Alianza del Pacífico.

En el mismo sentido, se crea en 2010 la Comunidad de Estados Latinoamericanos y Caribeños (CALC), la cual, más que un espacio económico, funciona de manera similar a la Unasur. La CALC es un espacio político, en el que se pretende profundizar la integración regional, fortalecer la cooperación en áreas de interés común y profundizar la integración política, económica y social de la región (CALC, 2010).

Por su parte, la Alianza del Pacífico es la más reciente iniciativa de integración regional. Es un área de integración profunda en la cual se pretende: "Avanzar hacia la libre circulación de bienes, servicios, capitales y personas, e impulsar un mayor crecimiento, desarrollo y competitividad de las economías de las partes" (Alianza del Pacífico, 2012, p. 1).

El simple hecho de realizar un listado como el anterior permite observar que no son pocos los esfuerzos enfocados en trabajar por la unidad de las economías de la región, lo cual indica en primera instancia que al parecer existe una voluntad política en todo el continente para darle vida a una amplia integración regional y, complementando con un aparte del profesor Briceño:

La integración debe dirigirse esencialmente [a] abolir las barreras y los obstáculos que impiden el libre flujo de bienes y servicios entre las diversas economías nacionales. De esta manera se lograría crear un espacio económico integrado en el cual la actuación de las leyes del mercado permitiría intensificar la interdependencia económica entre los países socios (Briceño, 2003, p. 31).

¿Cuál es entonces el obstáculo que no ha permitido realizar dicha integración? Es el interrogante que surge y, para tratar de darle respuesta, es necesario revisar algunos de los acuerdos de integración económica en América Latina.

En primer lugar, surgió la CAN, con el Acuerdo de Cartagena, en 1969. Más adelante nació el Mercosur, constituido en el Tratado de Asunción en 1991. Por último, y el más reciente intento por unir algunas de las economías de América Latina, es la Alianza del Pacífico, suscrita en 2010 en la Declaración de Lima.

Si bien no son los únicos acuerdos de integración en América Latina, debido al tamaño de sus economías, son los más relevantes en términos de población e ingreso: en la CAN viven cerca de $100 \mathrm{mi}$ llones de personas y el producto interno bruto de la región asciende a poco más de un billón de dólares; el Mercosur cuenta con una población de 286 millones de personas y su PIB ronda los 3.5 billones de dólares. Por su parte, en la Alianza del Pacífico viven alrededor de 207 millones de personas y su ingreso total es de 2.9 billones de dólares (datos elaborados por el autor a partir de las cifras actualizadas a 2012 en el Factbook de la Central Intelligence Agency.

$\mathrm{Si}$ bien la Comunidad Andina de $\mathrm{Na}$ ciones se planteó como uno de los primeros esfuerzos integracionistas de América Latina, diversos factores, como su estructura, el comportamiento de sus economías y la salida de uno de sus principales miembros, han implicado su fracaso a todo nivel.

En el estudio "¿Qué pasa con la Comunidad Andina de Naciones?" se 
establece que la CAN, como bloque subregional:

Es altamente inviable dadas las características inerciales del comportamiento y de las decisiones de sus miembros, las cuales han estancado el desarrollo del proceso, al punto que después de 37 años no se ha consolidado la etapa básica de la integración económica (Casas, 2007; Correa, 2007, p. 15).

Las economías nacionales de Colombia, Perú, Ecuador y Bolivia no han desarrollado el potencial de formar parte de un bloque subregional. Salvo un par de lineamientos políticos, como la iniciativa de los alcaldes de las zonas de frontera para fomentar la cooperación transfronteriza, y algunos encuentros de empresarios privados en la región, los resultados en materia de integración han dejado mucho que desear.

Por su parte, uno de los miembros que podía darle un mayor dinamismo a la economía andina, Venezuela, tomó la decisión de retirarse de la Comunidad y buscar su ingreso al Mercosur al mismo tiempo que conformaba la iniciativa del ALBA, esta última, una plataforma política regional para propagar el pensamiento socioeconómico del "Socialismo del siglo XXI". Con el reciente fallecimiento del presidente Hugo Chávez, debido a su carisma y al papel tan activo que desempeñaba en su proyecto bolivariano, hay que esperar para analizar el devenir del ALBA ante un nuevo mandatario en Venezuela.

Otra de las iniciativas de integración subregional es el Mercosur, el cual, a juicio del autor, ha sido hasta ahora la iniciativa de mejores resultados con relación a la integración de varias economías en la región.
Conformado por Brasil, Argentina, Uruguay, Venezuela (que fue recientemente reconocido como miembro), Paraguay (Estado sancionado y suspendido del Mercado debido al golpe de Estado al presidente Lugo) y Bolivia, en proceso de aceptación, el Mercosur surge como respuesta al fenómeno mundial de creación de zonas de libre comercio. La nueva tendencia en el comercio mundial es la liberalización por regiones, por medio de preferencias arancelarias, cuotas más generosas de importaciones y acuerdos sectoriales, además de negociaciones bilaterales para la resolución de conflictos comerciales. En el caso del Mercosur, si bien el comercio intrabloque es el que más aumenta, también se observa una tendencia creciente en los flujos desde y hacia el resto del mundo (Portela de Castro, s. f.).

El crecimiento de las economías del Mercosur ha sido positivo. De hecho, los datos están confirmando que se consolida la recuperación iniciada luego de la desaceleración ocasionada por la crisis financiera internacional (Lucángeli; Sanguinetti; Zamorano, 2011).

Las perspectivas de crecimiento para los países de la región son muy promisorias: "De acuerdo a la Cepal, la economía del Mercosur se expandiría por arriba del $7 \%$ para el año calendario, una tasa bastante más alta que la estimada para toda América Latina y el Caribe (5.2 \%)" (Cepal, 2010, p. 3).

Es claro que a nivel subregional el Mercosur es, de lejos, la mejor experiencia de integración económica, tanto así que, se percibe que puede ser alrededor del Mercosur que las demás economías se integren para darle una mayor fuerza y dinamismo a la economía latinoamericana.

En cuanto a las relaciones entre el Mercosur y la CAN, se encuentra: 
Un acuerdo entre el Mercosur y la CAN que elimine todas las barreras arancelarias es beneficioso para los países que integran ambos bloques. La liberalización arancelaria produce tanto un incremento en el PBI como en los volúmenes de comercio y en el nivel de empleo (Berrettoni; Cicowies, 2005, p. 7).

Por último, en cuanto a la Alianza del Pacífico, se puede decir que es la más reciente iniciativa de integración regional, lo cual es un hecho que demuestra el olvido de algunas de las economías latinoamericanas frente a la cuenca del Pacífico, que es la principal cuenca del comercio internacional, al integrar las economías de Asia, América y Oceanía.

La Alianza del Pacífico está conformada por Chile, Perú, Colombia y México. Todo parece indicar que Panamá será el quinto miembro del Acuerdo del Pacífico. Los orígenes de la Alianza del Pacífico pueden rastrearse hasta el Mercado Integrado Latinoamericano (MILA), una iniciativa de integración bursátil entre las Bolsas de Valores de Bogotá, Lima y Santiago. Si bien son dos espacios independientes, el MILA fue el primer paso que dieron las economías del Pacífico suramericano a favor de su integración.

Con tan solo un par de años de existencia, no es posible identificar un dinamismo positivo o negativo en materia económica en la Alianza del Pacífico. Sin embargo, hay un optimismo en general sobre sus consecuencias a nivel regional; de hecho, ya existen iniciativas de cooperación subregional en temas como la educación y la salud, al tiempo que la movilidad de los nacionales de los países miembro de la Alianza se ha visto favorecida al eliminar los visados para el turismo y los negocios.
Si bien en la Alianza se trabaja para fortalecer el comercio en su interior, no se ha presentado ninguna iniciativa de acercamiento con otras regiones de la cuenca del Pacífico, región natural de la Alianza. De hecho, tal y como lo describe la Cepal:

No existe suficiente conciencia acerca de la importancia del comercio y la inversión birregional y han existido pocas estrategias coordinadas entre países o agrupaciones regionales tendientes a estrechar los vínculos comerciales y de inversión con Asia Pacífico. El acercamiento de América Latina y el Caribe a esa región ha sido esporádico y poco sistemático, y se ha limitado a la conclusión de acuerdos bilaterales de libre comercio (Cepal, 2008, p. 11).

Solo hasta finales de la primera década del siglo XXI se comienza a hacer un esfuerzo multilateral del Pacífico latinoamericano de mirar hacia el Asia-Pacífico, y es en este contexto en el que surgen el ARCO y la AIP (Cepeda, 2012, p. 87).

Si no hay por parte de la Alianza del Pacífico una iniciativa de acercamiento con economías de otras regiones de la cuenca, tampoco existe un propósito para consolidar un proceso de integración a nivel de América Latina.

El país verde-amarillo se está posicionando como un líder regional y protagonista mundial. Ser miembro del G 20, del grupo BRIC, del foro IBSA, entre otros, hacen de Brasil el líder natural en su área de influencia y lo posicionan claramente como el país que dirige y establece el devenir del Mercosur, así como las políticas y prioridades temáticas.

Por su parte, en la Alianza del Pacífico no existe una clara dirigencia por 
parte de alguno de sus miembros, pero las economías más activas son la mexicana y la chilena, ambos países, con la intención de posicionarse como estrellas en la región.

Tanto Brasil como México y aun Chile, son países que desean atraer inversión extranjera a su territorio, $y$, debido a que una gran cantidad de los bienes y servicios de dichas economías compiten, en lugar de cooperar, es poco probable que desistan de su vanidad y sus pretensiones protagónicas para dar paso al encuentro de sus mercados y trabajar en procura de una integración más amplia.

De lo anterior surge otro interrogante que espera dar comienzo a un proyecto investigativo mucho más estructurado: ¿los procesos de integración subregional están contribuyendo, o por el contrario, son impedimentos para un proceso de integración de alcance regional?

\section{Conclusiones}

La voluntad existe, son muchas las iniciativas de integración regional, no solo en términos comerciales, sino también políticos, económicos y sociales. Sin embargo, se percibe cierta vanidad en los protagonistas de dichos procesos; al parecer, todos quieren liderar la integración suramericana y que los intereses y objetivos de sus naciones sobresalgan más allá de los demás.

Es muy pronto para concluir con contundencia este tema; por lo tanto, es necesario analizar con mayor detenimiento el desarrollo de la Alianza del Pacífico y el Mercosur como las dos principales estrategias integradoras de las economías de Latinoamérica.
El investigador, en este punto, plantea como sugerencia que las iniciativas de integración subregional están en el momento justo de comenzar a trabajar juntas, o por lo menos, coordinadas. Si se espera que cada una de las iniciativas avance por su cuenta, va a ser mucho más complejo en un futuro no muy lejano intentar acercarlas.

Por ejemplo, para atraer al principal elemento del Mercosur, Brasil, desde la Alianza del Pacífico, se pueden plantear las ventajas que traería para la economía carioca el contar con acceso a la cuenca de mayor crecimiento económico del planeta, y para los países del Pacífico, vincularse con el Mercosur traería enormes beneficios en términos de mercados potenciales.

A lo anterior debe sumarse el hecho de pensar en términos sinérgicos, en los que la suma de las partes en conjunto es mucho mayor que la suma de cada una de ellas, es decir, una gran economía latinoamericana, es mucho, mucho más influyente en el escenario global, que, como ha sucedido hasta ahora, una América Latina fragmentada.

Debido a que el más importante fenómeno que vive la humanidad en el presente siglo "es el de la profunda y creciente interdependencia que existe entre los países, las sociedades, las culturas y las economías de todo el mundo" (Moreno, 2007), es imperativo realizar una investigación, o darle vida a un observatorio, o semillero que, junto con la participación de los estudiantes de la institución, estudie la relación de estos procesos de integración subregional con el fin de establecer su impacto en una gran integración regional que comprenda la totalidad del territorio latinoamericano. 


\section{Referencias}

I. Acuerdo de Cartagena (1969). Acuerdo de Integración Subregional Andino.

2. Alianza Bolivariana para los Pueblos de Nuestra América, ALBA (2004). Declaración Conjunta para la creación del ALBA y el Acuerdo de aplicación del $A L B A$. Consultado el 16 de abril de 2013. Obtenido de http://www.alianzabolivariana.org/que_es_el_alba. php\#ancla1

3. Alianza del Pacífico (2012). Acuerdo Marco de la Alianza del Pacífico. Consultado el 16 de abril de 2013. Obtenido de http://www.fenalco.com.co/sites/ default/files/ acuerdomarco_0.pdf

4. Área de Libre Comercio de las Américas, ALCA (1994). Antecedentes del proceso del ALCA. Consultado el 16 de abril de 2013. Obtenido de http:// www.ftaa-alca.org/View_s.asp

5. Asociación de Estados del Caribe, AEC-ACS (1994). Convenio Constitutivo de la Asociación de Estados del Caribe. Consultado el 16 de abril de 2013. Obtenido de http://www.acs-aec.org/ es/documentos/legal/1994/convenioconstitutivo-de-la-asociacion-de-estados-del-caribe

6. Asociación Latinoamericana de Integración, Aladi (1980). Tratado de Montevideo de 1980. Consultado el 16 de abril de 2013. Obtenido de http:// www.aladi.org/nsfaladi/juridica.nsf/ vtratadoweb/tm80.

7. Asociación Latinoamericana de Libre Comercio, Alalc (1960). Tratado de Montevideo constitutivo de la Asociación Latinoamericana de Libre Comercio. Consultado el 16 de abril de 2013. Obtenido de http://www.parlamento.gub. uy/htmlstat/pl/tratados/ trat12859.htm
8. Berrettoni, D. y Cocowiez, M. (2005). El acuerdo de libre comercio MercosurComunidad Andina de Naciones: una evaluación cuantitativa. Santiago de Chile, Chile: Centro de Proyecciones Económicas, División de Estadística y Proyecciones Económicas.

9. Briceño, J. (2003). Las teorias de la integración regional. Mérida, Venezuela: Ediciones del Vicerrectorado Académico de la Universidad de los Andes.

ıo. Caetano, G. (2011). Mercosur: 20 años. Montevideo, Uruguay: Cefir.

I I. Casas, A. y Correa, M. E. (2007). ¿Qué pasa con la Comunidad Andina de Naciones? Papel Politico, 12(2), 591-632.

I2. Cepeda, J. C. (2012). Cooperación e integración regional en América Latina: el Foro del arco del Pacífico latinoamericano y la Alianza del Pacífico. ¿Cómo Vamos?, III, 86-93.

I3. CIA (2012). The World factbook. Consultado el 16 de abril de 2013. Obtenido de https://www.cia.gov/library/publications/the-world-factbook/

I4. Comisión Económica para América Latina y el Caribe, Cepal (1987). Raúl Prebisch: un aporte al estudio de su pensamiento. Santiago de Chile, Chile: Cepal.

I5. Comisión Económica para América Latina y el Caribe, Cepal (2008). El arco del Pacifico latinoamericano y su proyección a Asia-Pacífico. Santiago de Chile, Chile: Naciones Unidas.

i6. Comisión Económica para América Latina y el Caribe, Cepal (2010). Estudio económico de América Latina y el Caribe. 2009-2010. Santiago de Chile, Chile: Naciones Unidas.

17. Comunidad Andina de Naciones, CAN (1969). Acuerdo de Integración Subregional Andino. Consultado el 16 de abril de 2013. Obtenido de http:// 
badicc.eclac.cl/ controversias/Normativas/CAN/Espanol/Acuerdo_de_Cartagena.pdf

I8. Comunidad de Estados Latinoamericanos y Caribeños, CALC (2010). Cumbre de la Unidad de América Latina y el Caribe. Consultado el 16 de abril de 2013. Obtenido de http://www.diputados.gob.mx/cedia/sia/spe/SPE-CIA-03-10.pdf

19. Comunidad del Caribe, Caricom (1973). Revised treaty of Chaguaramas establishing the Caribbean Community including the Caricom single market and economy. Consultado el 16 de abril de 2013. Obtenido de http://www.caricom.org/jsp/ community/revised_treaty-text.pdf

20. Declaración de Lima (2011). Declaración Presidencial sobre la Alianza del Pacífico para la conformación de un Área de Integración Profunda (AIP).

21. Gudynas, E. (2007). Integración y comercio. Diccionario latinoamericano de términos y conceptos. Montevideo, Uruguay: Claes y Oxfam.

22. Hu, J. (noviembre 12 de 2004). Joining Hands to Enhance Friendship between China and Latin America. Speech by Chinese President Hu Jintao at the Brazilian Parliament.

23. Lucángeli, J.; Sanguinetti, M. y Zamorano, A. L. (2011). Mercosur: la consolidación de la expansión de la economía del bloque. Buenos Aires: Ministerio de Relaciones Exteriores y Culto de la República Argentina.

24. Moreno, C. (enero-junio de 2007). Integración latinoamericana: ALCA vs. ALBA. Presente y pasado. Revista de Historia, 23, 155-178.

25. Observador Global.com (2012). Los objetivos de la nueva Alianza del Pacífico.
26. Pizarro, R. (2008). El difícil camino de la integración regional. Nueva Sociedad, 214.

27. Portela de Castro (s. f.). Realidades y desafios de las negociaciones externas del Mercosur. Coordinadora de Centrales Sindicales del Cono Sur.

28. Prebisch, R. (1959). El mercado común latinoamericano. Santiago de Chile, Chile: Cepal.

29. Sistema Económico Latinoamericano y del Caribe, SELA (1975). Convenio de Panamá Constitutivo del Sistema Económico Latinoamericano (SELA). Consultado el 16 de abril de 2013. Obtenido de http://216.122.62.22/attach/258/ default/T023600000397-0-Convenio_de_Panama_(enero_2006).pdf

30. Tratado de Asunción (1991). Tratado para la constitución de un mercado común entre la República Argentina, la República Federativa del Brasil, la República del Paraguay y la República Oriental del Uruguay. Consultado el 16 de abril de 2013. Obtenido de http: //idatd.eclac.cl/controversias/Normativas/MERCOSUR/Espanol/Tratado_ de_Asuncion.pdf

3I. Unión de Naciones Suramericanas, Unasur (2008). Tratado Constitutivo de la Unión de Naciones Sudamericanas. Consultado el 16 de abril de 2013. Obtenido de http://www. comunidadandina.org/unasur/tratado_constitutivo. htm

32. Vieira, E. (2005). Evolución de las teorías sobre integración en el contexto de las teorías de relaciones internacionales. Papel Politico, 18, 235-290. 